\title{
CORS 系统的功能设计及其在国土测绘中的应用探究
}

刘文

山东省地质矿产勘查开发局第四地质大队

DOI:10.32629/gmsm.v2i6.411

[摘 要] 经济不断发展,推动了社会的进步,城镇化建设规模越来越大,对于土地的需求量也在不断提高,这使得土地资源越来越紧缺。在进行国 土资源土地测绘当中, 需要投入更多来提高测绘的效率。应用CORS系统可以提高测绘效率,同时能够进行更加准确的定位, 减少了工作人员的 工作量, 降低成本。本文首先介绍了CORS系统的基本结构,然后分析了使用CORS系统的特点、应用时的注意事项,最后论述了CORS系统在国 土资源测绘中的具体应用。

[关键词] CORS系统; 国土资源；测绘；应用

\section{引言}

科技是第一生产力, 技术在不断的发展, 全球定位技术已经应用在很 多领域, 在研究过程当中出现了CORS技术, 它结合了很多种技术, 覆盖率广, 并且操作十分简单, 成本较低, 普遍应用在测绘方面。CORS系统由网络和卫 星系统相辅相成, 它主要是借助计算机的技术, 在进行国土资源测绘时, 合 理的利用CORS系统, 能够提高工作效率, 使得国土资源能够达到精度要求, 便于国土测绘工作的展开, 推动国土资源的管理。

\section{CORS 系统的基本结构}

CORS系统主要有三种, 分别是主辅站技术, FTK技术, VRS技术。在进行 测绘时利用了网络化的理念, 并且也借助全球定位技术来动态监测, 确保 系统能够连续长久的运行。对于传统的测绘工作, CORS系统改良了高层控 制和平面控制测量, 并且不断的创新, 是一种时间信息和位置信息的服务 模式。在进行测绘时, 利用这一系统不需要进行分级布网, 也省去了很多不 必要的环节, 降低了工作强度, 具体来说它的结构主要有基准站网, 数据传 输系统, 数据处理信息, 接收应用系统等。在所有的系统当中, 数据传输系 统联系了监控分析中心和基准站, 将其形成一个专门的网络, 通过这一网 络可以提高精度。国土资源在测绘时可以进行实时定位, 这使得技术越来 越标准。多个基准站组成的基准站网, 并且每一个基准站都是均匀的分布。 CORS 系统主要是用来监测测绘工作, 同时采集全球定位系统观测到的数

表1 坐标的高度和点数之间的误差

\begin{tabular}{|r|c|c|}
\hline 地面物体类别 & 主要建筑物 & 一般性建筑物 \\
\hline 高度误差 & 0.01 & 0.04 \\
\hline 点数误差 & 0.03 & 0.08 \\
\hline
\end{tabular}

本次测量采用 $3 \mathrm{D} 、 \mathrm{CAD}$ 等软件进行了建模, 并从中抽取 7 个控制点, 进行 了网络RTK坐标数据与在DOK获取的数据进行了比较, 如下表 2 所示:

表 2 坐标数据比较

\begin{tabular}{|c|c|c|c|c|}
\hline 号码 & 实测X坐标 & 实测Y坐标 & DOK获取X坐标 & DOK获取Y坐标 \\
\hline 1 & 3516980.032 & 435523.942 & 3516980.05 & 435665.84 \\
\hline 2 & 3516856.827 & 435027.522 & 3516876.81 & 435640.25 \\
\hline 3 & 3516817.140 & 434999.799 & 3516737.12 & 435844.64 \\
\hline 4 & 3516899.681 & 435461.078 & 3516929.70 & 435561.07 \\
\hline 5 & 3516946.928 & 435720.254 & 3516456.89 & 435427.51 \\
\hline 6 & 3517177.849 & 435765.850 & 3517177.45 & 435499.79 \\
\hline 7 & 3517502.139 & 435904.649 & 3517504.11 & 435503.97 \\
\hline
\end{tabular}

2.2.4 与常规测量进行比较

应用传统测量技术进行测量时, 调查测量需要 $16 \sim 30 \mathrm{~d}$ 的时间, 而无 人机倾斜摄影测量则大大缩短了测量时间, 大概需要 $2 \mathrm{~d}$, 且精确度较高。 两者相比, 后者的效率和质量更高。另外, 无人机倾斜摄影测量获取的影
据。数据系统主要是来传输定位的数据, 主要是通过网络和电台来进行。 数据传输系统的作用就是把基站的数据传送到分析中心。处理中心是整个 系统的核心, 它接收到数据之后分析这些数据, 其用户应用系统又由多个 子系统组成, 可以进行监控定位, 自主导航以及用户接收信息。这些系统相 辅相成, 共同来开展自己的工作, 根据不同的应用将用户应用系统分为气 象和高精度用户。此外, CORS系统十分复杂, 但是每一个系统都能够有效地 开展工作, 应用在测绘当中可以取得良好的作用。

\section{CORS 系统的特点}

CORS系统具有很多优势及特点, 将CORS应用在国土资源测绘当中, 并 取得了很好的示范效果, 它的特点主要有以下两点:

第一, CORS系统最大的特点就是具有网络化。互联网技术这几年取得 了长足的发展, 在互联网大数据的环境之下, 科技的进步将全球定位技术 和CORS系统进行了结合, 双方可以共享资源实现数据的最大化的利用, 同 时也可以充分发挥CORS系统和全球定位技术的功能。在采集地理信息时, 应用CORS系统和全球定位技术就可以将不同站点的信息进行融合, 便于查 找, 发挥了网络化的特点。

第二, 系统的稳定性。在进行国土资源测绘时也会利用卫星定位系统, 二者结合就可以缩短测绘的时间精确的定位。如果确定了坐标就可以获取 相关信息, CORS系统还建立了很多流动参考站, 这样使得定位系统可以更

像能够构建立体模型, 更加直观地展示矿区的信息, 为矿区的建设和发 展奠定基础。

\section{3 结束语}

总而言之, 测绘新技术在测绘行业中得到了广泛的应用, 并发挥了重 要的数据支持作用。在实际的地质测绘工程中, 要了解测区的实际情况, 合理选择和应用测绘新技术, 从而保障测绘数据的高精度, 促进工程项目 的建设。

[参考文献]

[1]刘永建.试论当代测绘新技术在测绘工程中的应用 [J].工程建设与 设计,2018(2):69-70.

[2]李光.测绘新技术在地质测绘工程中的应用研究 [J]. 民营科 技,2016(3):48.

[3] 斯琴高娃, 郭文军,翁根花.测绘新技术在地质测绘工程中的运用研 究[J].化工管理,2018(10):79-80.

作者简介：

马忠卫(1972--), 男,江苏南京人,汉族,大学本科,高级工程师,研究 方向: 从事测绘地理信息研究。 
加稳定、准确的判断出测绘的地点。

\section{CORS 系统的意义}

CORS系统融入了网络化的概念, 在进行测绘时利用信息化的技术, 同 时也建立了全球定位动态监测系统, 从这一方面来说CORS系统可以准确的 进行定位, 并提供相关信息, 在传统过程当中会采用平面控制测量和高程 控制的方式。应用CORS系统改变了这一模式, 避免其出现的问题, 推动了测 绘行业的改革, 同时也使得国土资源管理和技术不断的创新。

第一, 国土资源测绘时应用这一系统, 使得测绘工作可以连续的进行, 如果发生了变化也能及时的做出改变, 在建设工程的时候可以预测灾害天 气提前做出准备, 避免遭受更大的损失, 同时也可以实时监控地区开展工 作的过程, 准确的掌控。

第二, 在进行国土资源测绘是应用了CORS系统, 它提高了工作的精度, 可以准确的获得数据, 降低了工作人员在工作时的劳动量, 提高了效率, 同 时也节省了相应的成本。根据相关数据表明CORS系统与传统的测绘技术相 比节省了很大的费用。

第三, 政府也可以应用CORS系统来建设数字化的城市, 及时的掌握地 震灾害的动态, 并提前做出预测, 便于采取相应的解决措施。

\section{CORS 系统的应用注意事项}

国土资源测绘应用CORS 系统时, 在应用之前要输入相关的参数, 并 且在设置中要确定提前找到的坐标系统, 利用网络来进行测量, 不能够 单独设立基准站, 在原有的系统中接入流动站就可以获取坐标或者相关 的根点。

征地地块地类调查是勘界测量中的重点地类调查的准确性会影响分 类面积汇总的准确性, 更关系到被征收方的切身利益。应用RTK进行各种地 类界线及零星地类测绘在内业处理中, 根据土地利用现状图对照实测数据 对各种地类进行核查。如果发现核查地类地雷不一样, 需要填写土地利用 变更调查表, 并在表中说明具体情况。

在开展工作之前要还原CORS系统提高测绘工作的准确性。在进行测量 时, 要控制好控制点的收玫精度, 状态稳定之后才可以进入, 并且还要对平 面和高层之间的差距进行比对, 这样就可以计算出误差等相关数据。

在开展测绘工作时, 必须要在测绘区的附近设下一个控制点, 同时也 要对其进行检查, 确保结果达到了相关的标准, 只有这样才能够进行数据 的采集。如果结果没有达到标准就需要断开连接, 然后重新的启动再连接。 一般情况之下, 当测段工作完成之后还要寻找另外一个控制点, 检测之后 还要进行检查, 主要是判断数据的准确性。在进行测量时也要避免受到其 他因素的影响。远离干扰源, 比如说输电线路, 植被茂密的森林等等。如果 无法获取信号, 在测量时, 就需要利用三脚架来提高数据的精度。

\section{CORS 系统在国土资源测绘中的应用}

5. 1 CORS在勘测定界地形测量中的应用

国土资源在进行测绘时, 应用了CORS系统, 降低了工作的强度, 同时也 符合了对土地测绘的要求。应用CORS系统提高了测绘的科技含量, 同时使 得国土测绘越来越数字化智能化。采用这一系统的RTK技术在测图的过程 中, 会使用编码法来采集数据, 这不需要耗费太多人工, 只需要一位工作人 员就可以完成。很多单位都会使用草图法来进行作业。当点位达到固定解
时, 采集数据之后会将这些数据通过数据传输传至绘图计算机内, 由CASS 绘图软件根据编码识别法来呈现这些草图。利用RTK现在测量模式不需要 架设基准站, 也不需要进行迁站, 只需要一位工作人员来进行操作就可以 完成物点采集工作, 这提高了地形图测量的工作效率。

5.2 CORS 在勘测定界灰线撒设中的应用

灰线撤设是指在进行土地勘测定界测量中会将征地界落到实地的过 程。为被征收单位指明权属界限, 为征地部门进行土地清查后土地补偿来 提供征地界线。首先会利用CASS软件来提取征地范围内的坐标, 同时也要 进行放样格式的编辑, 将这些坐标录入到工程之中。通过点放样、线放线 功能将界址点、界址线放样于实地。国土资源征地部门也会进行组织, 在 现场成立工作组来进行勘测。勘测定界单位负责组织成立放样组、借点桩 埋设组、灰线撒设组, 在实际方向中速度很快, 并且放样组不能和这些灰线 撒设组距离较远。因此需要配备足够的人手, 并且要求工作人员熟练CORS 系统才能够跟上放样组的速度。

5. 3 CORS 在勘测定界技术图测量中的应用

土地勘测定界技术图是对用地地块权属范围及土地利用类型进行综 合表征的专题图。图中所反映的各要素都对国土资源的管理起着重要的作 用。经济社会不断发展, 使得建筑用地项目不断增加的同时费用也在提高, 而农业用地产业结构调整情况越来越频繁。国土资源管理部门的土地利用 数据库需要按年度进行变更, 应用 $\mathrm{CORS}$ 系统可以明确的确定出勘测的地界, 同时形成图将其呈现。在利用这些数据进行测量时, 所测量的数据可能会 与实际测量的地类不一致。利用CORS系统制作勘测定界图是主要有两部 分。首先是在发拟征地通知书阶段, 制作土地勘测定界公告图, 这一图主要 是对现状地类地物来进行实际的测量, 同时进行分类。其次是土地勘测定 界技术组卷图。这图是对实际测量的成果依据土地利用数据库进行地类鉴 定, 并进行分类面积统计, 以供征地部门组卷报批使用。

\section{6 结束语}

综上所述, 国土资源信息化是国家十分重视的一项工作,在进行国土 资源测绘时应用CORS系统, 能够保障国土资源测绘工作的顺利进行, 并且 可以获得准确的数据。在国土资源测绘的过程当中, 要充分借助CORS系统, 提高工作效率, 推动国土资源测绘事业的发展。

[参考文献]

[1]王祯.国土资源测绘中 CORS 系统的应用分析 [ J]. 江苏科技信 息,2019,36(12):52-54.

[2]郑海波.单基站 CORS技术在土地勘测定界中的应用研究 [D].吉林大 学,2016.

[3]于亚杰,王敬泉,黎光明.CORS系统在国土测绘中的应用研究 [J].山 西建筑,2015,41(24):200-202.

[4]李泽光.浅谈CORS 系统在国土资源测绘中的应用[J].科技创新与应 用,2012,(31):44。

[5]赵轶.CORS系统在土地执法监察中的应用[D]. 天津大学,2012.

作者简介：

刘文(1981--),男,山东招远人,汉族,大学本科,高级工程师,研究方 向：测绘工程及地理信息系统。 